\title{
Beauty Production with the ZEUS HERA II Data
}

Oliver Maria Kind

- On Behalf of the ZEUS Collaboration -

Physikalisches Institut

der

Rheinischen Friedrich-Wilhelms-Universität Bonn

XIV International Workshop on Deep Inelastic Scattering

KEK - Tsukuba, Japan

April 22, 2006
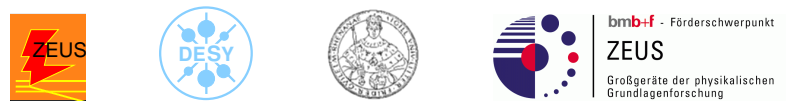


\section{Outline}

Introduction

HERA II Running

Motivation

Beauty Extraction Methods

Previous Results

Beauty in Photoproduction

Dijet PhP Event Selection

Beauty Extraction

Results

Beauty in Deep Inelastic Scattering

Event Selection

Control Plots

Beauty Extraction by $p_{\perp}^{\text {rel }}$

Results

Summary 


\section{ZEUS and HERA II Running}
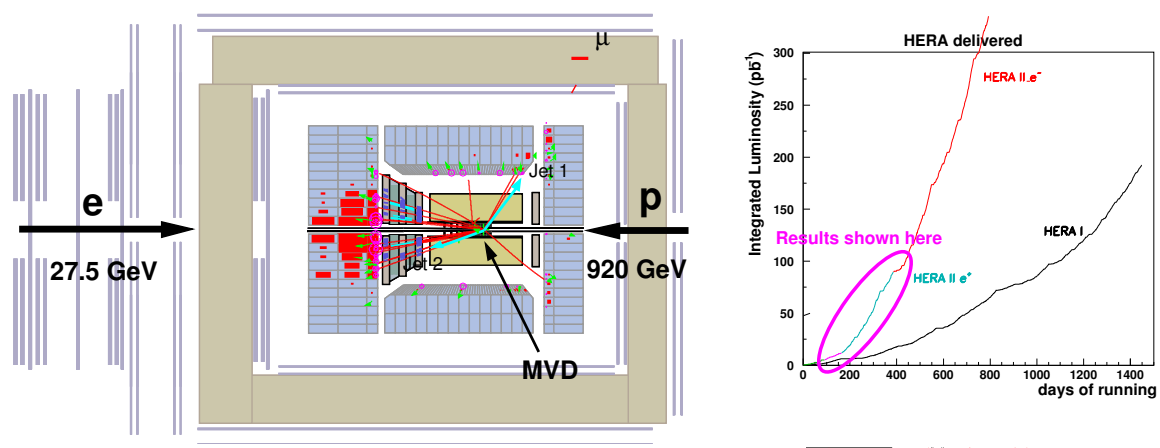

- HERA II upgrade: large increase of luminosity

- ZEUS micro-vertex detector taking data since 2003 ( $\rightarrow$ life-time tag)

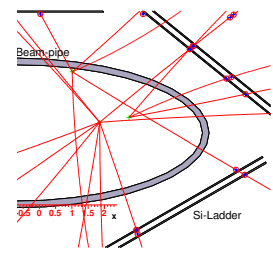




\section{Motivation}

- Beauty production at HERA is good testing ground for PQCD

- Multiple scales: $m_{\mathrm{b}}, p_{\perp}^{\mathrm{b}}$ and $Q^{2}$

- Probe the $b$ contribution to the proton structure function, $F_{2}^{\mathrm{b} \bar{b}}$

- PYTHIA MC: LO + PS (includes flavour excitation diagrams)

- NLO QCD calculations available (FMNR, HVQDIS)
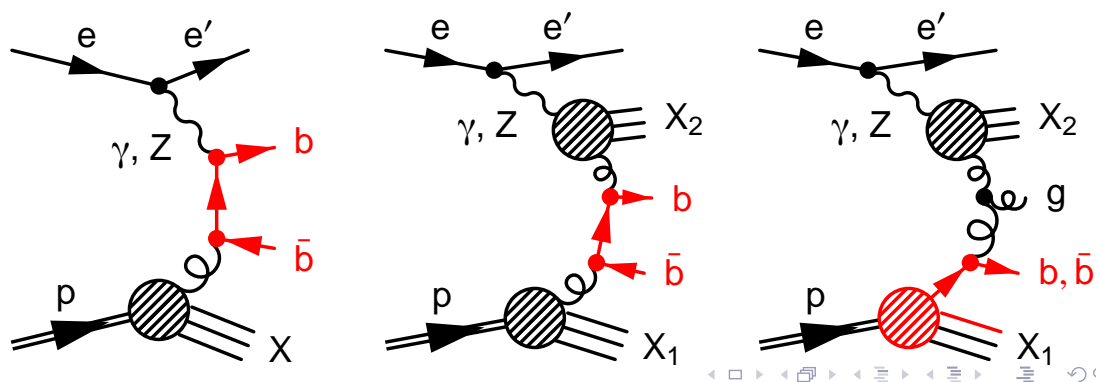


\section{Beauty Extraction Methods}

Component of $\mu$ momentum transverse to jet axis, $p_{\perp}^{\text {rel }}$

- Large for $B$ decays because of large $B$ mass

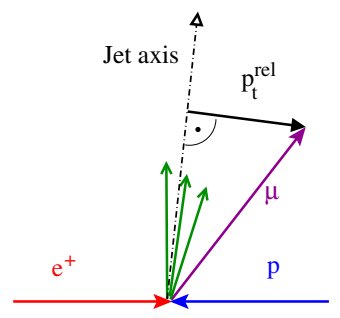

Signed $\mu$ impact parameter, $\delta$

- Symmetrically distributed around zero for light flavours

- Positive tail for beauty and charm due to life-time

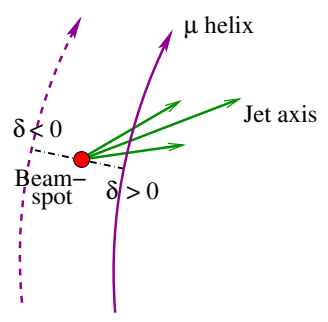




\section{Previous Results}

Situation:

- Most measurements agree with NLO QCD within $2 \sigma$

- Overall, NLO tends to somewhat underestimate the data especially towards low $p_{\perp} \rightarrow$ investigate

- Statistics still low

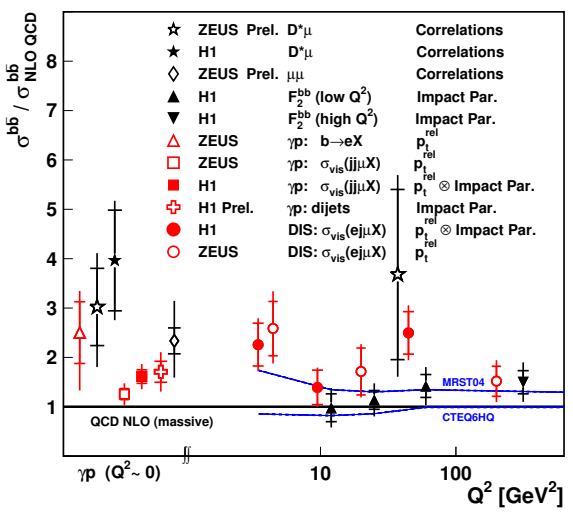

Aim of HERA II:

- Increase statistics $\rightarrow$ single- and double-differential $x$-sections

- Reduce systematics by complementary measurements (B life-time) 


\section{Part I}

\section{Beauty in Photoproduction}

$4 \square>4 \square>4 \equiv>4 \equiv$ 


\section{Dijet $\mathrm{PhP}+\mu$ Event Selection}

$2004 \mathrm{e}^{+} p$ data: $\mathcal{L}=33 \mathrm{pb}^{-1}$

Photoproduction:

- Veto on scattered $\mathrm{e}^{+}$

- $0.2<y_{\mathrm{JB}}<0.8$

Jet finding:

- $k_{\perp}$-clustering

- $N_{\text {jets }} \geq 2$

- $p_{\perp}>7(6) \mathrm{GeV}$

- $|\eta|<2.5$

\section{Associated $\mu$ :}

- $\mu$ inside jet

- $p_{\perp}^{\mu}>2.5 \mathrm{GeV}$

- $-1.6<\eta^{\mu}<2.3$

- $\mu$-chambers + central tracking 


\section{Dijet $\mathrm{PhP}+\mu$ Event Selection}

Events selected: $\approx 1800$

Signal:

- $\mu$ from SL decays of $b$ and $\mathrm{C}$

Background:

- Fake- $\mu$ from punch-through and in-flight decays from $\pi, \mathrm{K}$

Shapes reproduced by PYTHIA 6.2

\section{ZEUS}
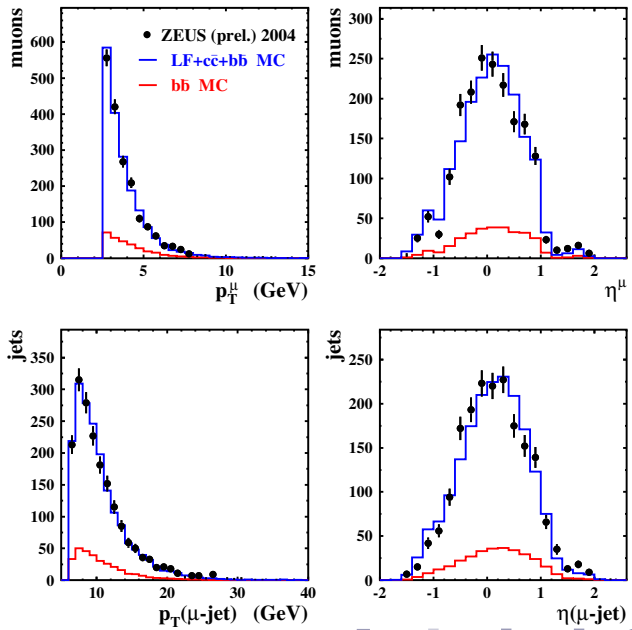


\section{Beauty Extraction}

- Combined fit of $p_{\perp}^{\text {rel }}$ and impact parameter, $\delta$
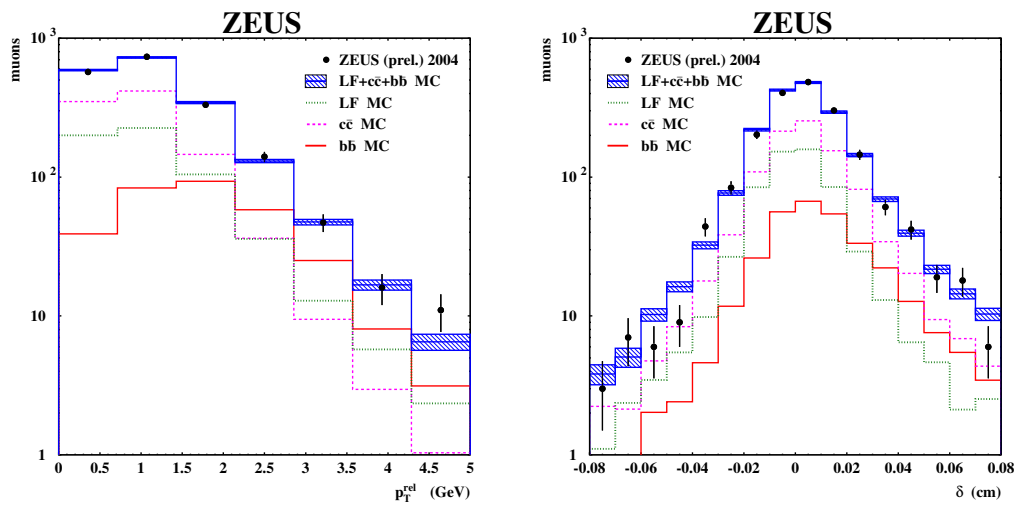


\section{Beauty Extraction}

Result:

$f_{\mathrm{b}}=(16.7 \pm 2.6) \% \quad f_{\mathrm{c}}=(52 \pm 10) \%$

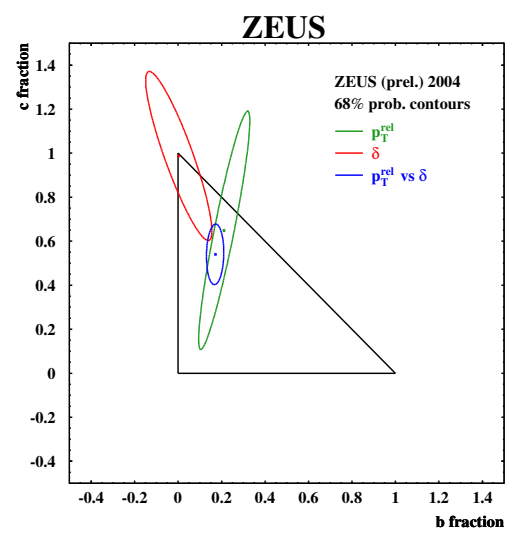

Remarks:

- PYTHIA 6.2 templates used for b, $c$ and light-flavours

- $p_{\perp}^{\text {rel }}$ shape of light-flavour MC corrected by inclusive dijet data

- Beam position measured run-by-run

- Resolution of $\delta$ in MC modeled on inclusive data (latest MVD alignment not in) 


\section{Results for $\mathrm{e}^{+} \mathrm{p} \rightarrow \mathrm{e}^{+\prime}+\mathrm{b} \bar{b}+\mathrm{X} \rightarrow \mathrm{e}^{+^{\prime}}+\operatorname{dijet}+\mu+\mathrm{X}$}

Kinematic region:
$Q^{2}<1 \mathrm{GeV}^{2}$
$0.2<y<0.8$
$p_{\perp}^{\text {jet }}>7(6) \mathrm{GeV}$ $\left|\eta^{\mathrm{jet}}\right|<2.5$
$p_{\perp}^{\bar{u}}>2.5 \mathrm{GeV}$
$-1.6<\eta^{\mu}<2.3$

Conclusions:

- Agreement with NLO QCD prediction (FMNR)

- Agreement with ZEUS data from HERA I running

- $p_{\perp}^{\text {rel }}$ only

- $\approx 3 \times$ statistics than '04 analysis

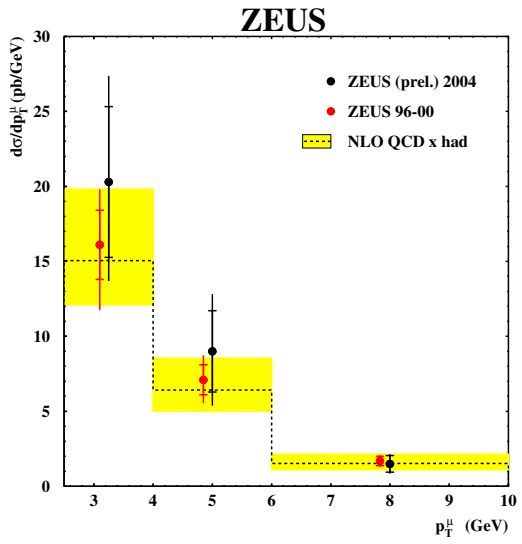




\section{Part II}

\section{Beauty in Deep Inelastic Scattering}




\section{Event Selection: $\mathrm{ep} \rightarrow \mathrm{e}^{\prime}+\mathrm{b} \overline{\mathrm{b}}+\mathrm{X} \rightarrow \mathrm{e}^{\prime}+$ jet $+\mu+\mathrm{X}$}

2003/4 ep data: $\mathcal{L}=39 \mathrm{pb}^{-1}$

DIS:

- $Q^{2}>4 \mathrm{GeV}^{2}$

- $E_{\mathrm{e}^{\prime}}>10 \mathrm{GeV}$

- $y_{\mathrm{el}}<0.7$

- $40<\left(E-p_{z}\right)<65 \mathrm{GeV}$

Jet finding:

- $k_{\perp}$-clustering

- $\geq 1$ jet with associated $\mu$

- $E_{\perp}>5 \mathrm{GeV}$

- $-2.0<\eta<2.5$
Associated $\mu$ :

- $\mu$ inside jet

- $p_{\perp}^{\mu}>1.5 \mathrm{GeV}$

- $\mu$-chambers + inner tracking

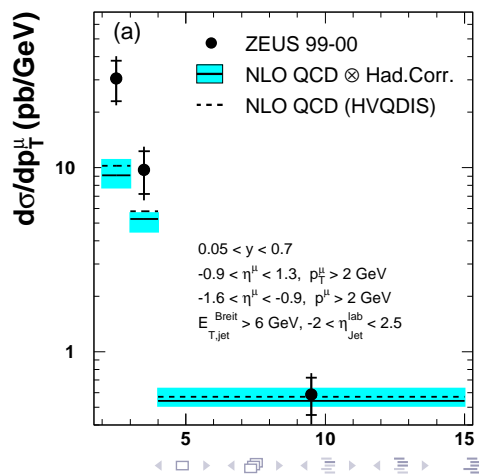




\section{Event Selection: $\mathrm{ep} \rightarrow \mathrm{e}^{\prime}+\mathrm{b} \overline{\mathrm{b}}+\mathrm{X} \rightarrow \mathrm{e}^{\prime}+$ jet $+\mu+\mathrm{X}$}

2003/4 ep data: $\mathcal{L}=39 \mathrm{pb}^{-1}$

DIS:

- $Q^{2}>4 \mathrm{GeV}^{2}$

- $E_{\mathrm{e}^{\prime}}>10 \mathrm{GeV}$

- $y_{\mathrm{el}}<0.7$

- $40<\left(E-p_{z}\right)<65 \mathrm{GeV}$

Jet finding:

- $k_{\perp}$-clustering

- $\geq 1$ jet with associated $\mu$

- $E_{\perp}>5 \mathrm{GeV}$

- $-2.0<\eta<2.5$
Associated $\mu$ :

- $\mu$ inside jet

- $p_{\perp}^{\mu}>1.5 \mathrm{GeV}$

- $\mu$-chambers + inner tracking

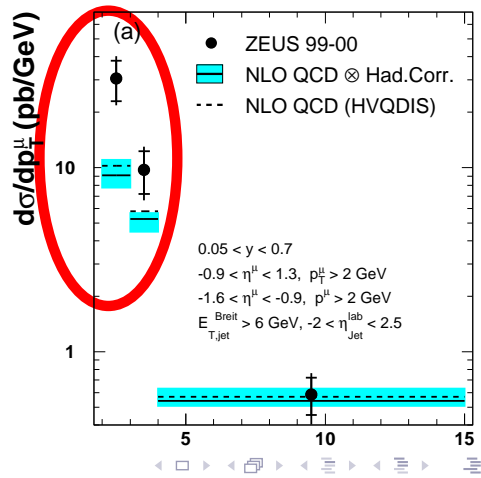




\section{Control Plots}
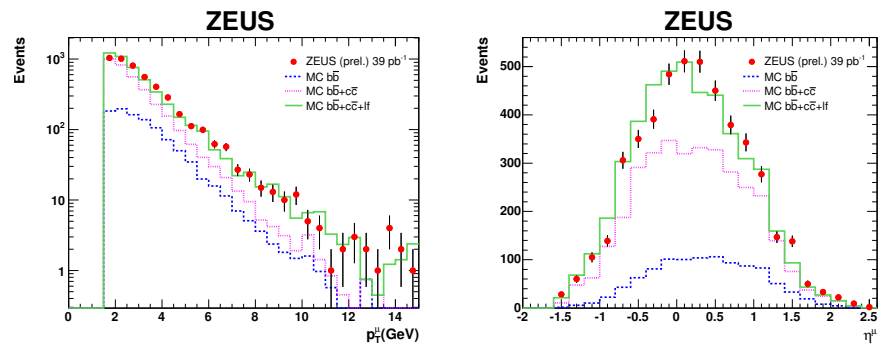

\section{ZEUS}
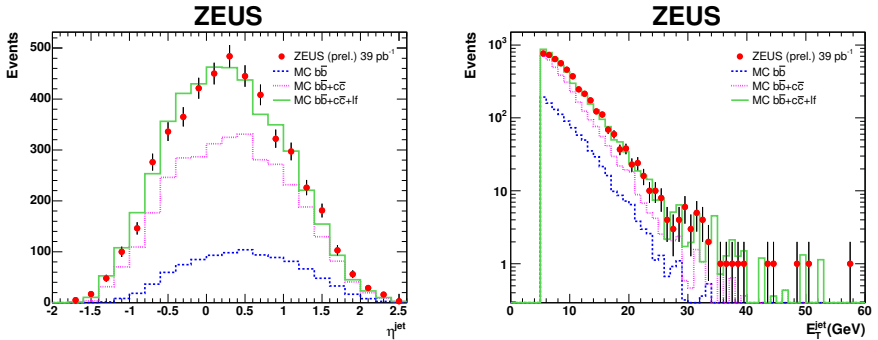


\section{Beauty Extraction by $p_{\perp}^{\text {rel }}$}

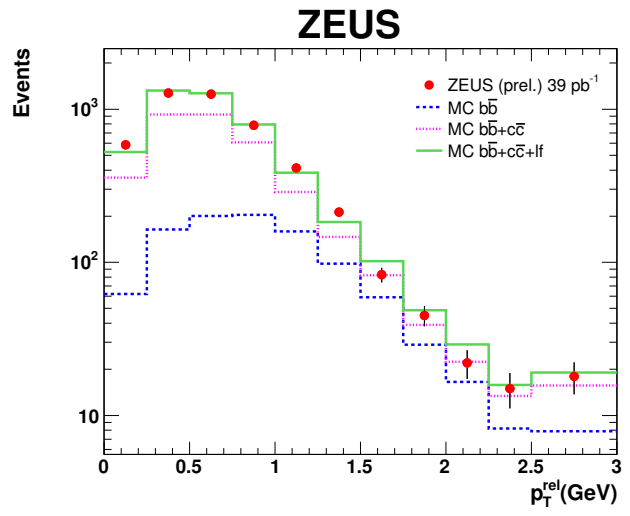

- Fit result: $f_{b}=(21.4 \pm 2.1) \% \quad$ i.e. $\quad \gtrsim 1000 \mathrm{~b}$ events $/ 39 \mathrm{pb}^{-1}$

- K-factor for Beauty LO+PS MC (RAPGAP) of 2.49

- Impact parameter analysis ongoing 


\section{Results for ep $\rightarrow \mathrm{e}^{\prime}+\mathrm{b} \overline{\mathrm{b}}+\mathrm{X} \rightarrow \mathrm{e}^{\prime}+$ jet $+\mu+\mathrm{X}$}

\section{ZEUS}

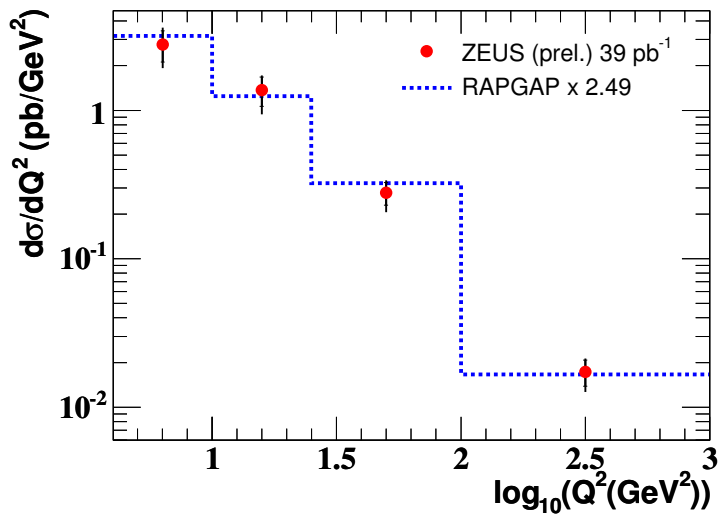

Kinematic region:

- $Q^{2}>4 \mathrm{GeV}^{2}$ $0.05<y<0.7$

- $E_{\perp}^{\text {jet,lab }}>5 \mathrm{GeV}$ $-2<\eta^{\text {jet }}<2.5$

- $p_{\perp}^{\mu}>1.5 \mathrm{GeV}$ $\eta^{\mu}>-1.6$

Result: $\quad \sigma_{\mathrm{b} \bar{b}}=\left(77.1 \pm 7.8_{-14.9}^{+9.6}\right) \mathrm{pb}$

NLO predictions not yet calculated (in progress) 


\section{Results for ep $\rightarrow e^{\prime}+b \bar{b}+X \rightarrow e^{\prime}+$ jet $+\mu+X$}

\section{ZEUS}

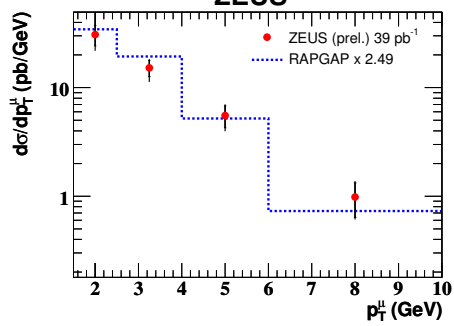

ZEUS

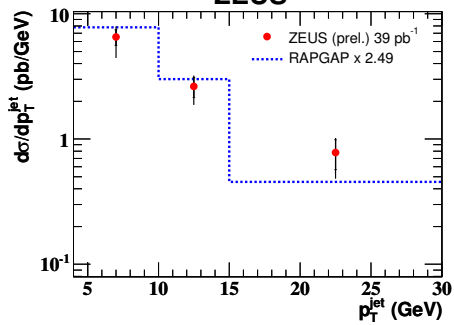

\section{ZEUS}

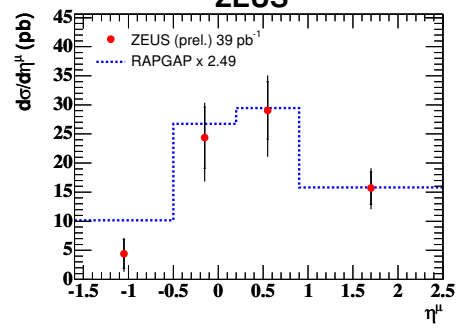

\section{ZEUS}

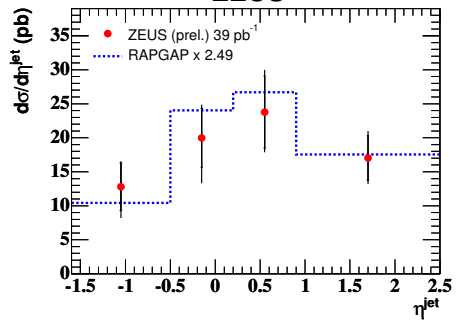




\section{Results for ep $\rightarrow e^{\prime}+b \bar{b}+X \rightarrow e^{\prime}+$ jet $+\mu+X$}

\section{ZEUS}

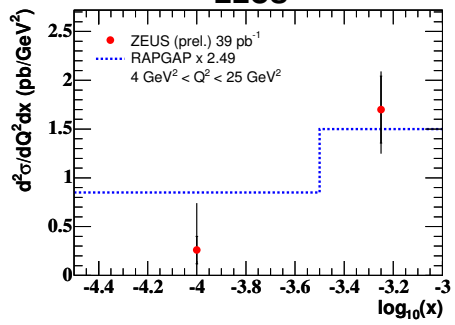

ZEUS

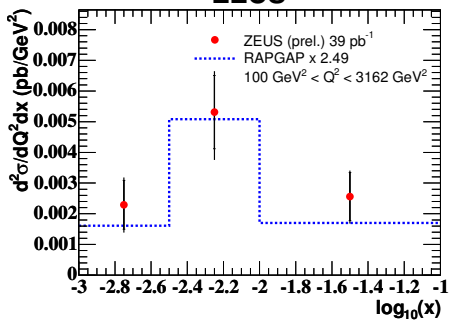

\section{ZEUS}

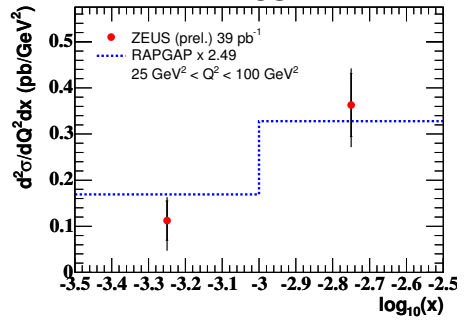

double-differential $x$-sections in $x$ and $Q^{2}$ 


\section{Summary}

$\mathrm{PhP}$ analysis:

- $1^{\text {st }}$ B analysis @ZEUS exploiting the MVD

- B extraction by combining $p_{\perp}^{\text {rel }}$ and impact parameter

- Results consistent with NLO QCD and HERA I measurements

DIS analysis:

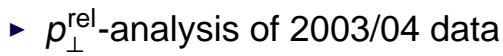

- Results consistent with LO+PS MC shapes

- NLO calculations in progress

- Looking forward to $F_{2}^{\mathrm{b} \bar{b}}$ measurement 


\section{Part IV}

\section{Back-up Slides}

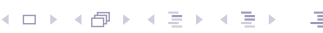




\section{Event Selection in Detail}

Pre-selection:

- All good events

- Trigger on

- jets in PhP

- SL $\mu$ in PhP

- jets $+\mu$

Vertex:

- $-40<Z_{\mathrm{vtx}}<4 \mathrm{~cm}$

Tracking:

- $\geq 2$ vtx tracks

- $\frac{\text { No. of all tracks }}{\text { No. of vtx tracks }} \leq 10$
Veto on $\mathrm{e}^{\prime}$ :

- $\mathcal{P}>0.9$

- $E_{\mathrm{e}}>5 \mathrm{GeV} \wedge y_{\mathrm{el}}<0.9$

EFOs:

- $0.2<y_{\mathrm{JB}}<0.8$

Calorimetry:

- $E_{\perp}-2$ inner rings $\geq 10 \mathrm{GeV}$

- $p_{\perp} / E_{\perp}<0.5$ 


\section{Event Selection in Detail}

Jets:

- $\geq 2$ jets found with $k_{\perp}$-clustering in $E$ recombination scheme (massive mode, 3211) and

- $p_{\perp}>7(6) \mathrm{GeV} \wedge|\eta|<2.5$

$\mu$ finding:

- $p_{\perp}^{\mu}>2.5 \mathrm{GeV}$

- $-1.6<\eta<2.3$ 


\section{Previous Results}
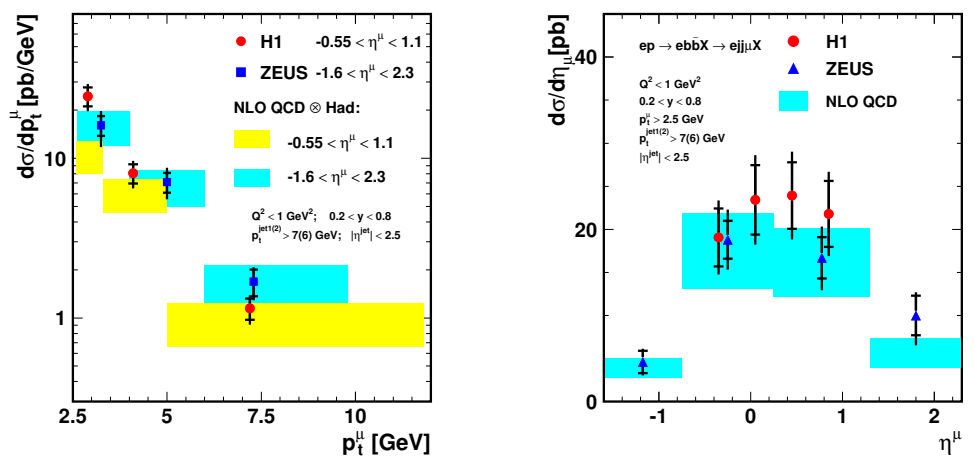


\section{Data Sets}

\begin{tabular}{lrr}
\hline Set & & $\mathcal{L}$ \\
\hline \hline ZEUS 2003/04 data & & $39 \mathrm{pb}^{-1}$ \\
Beauty MC & RAPGAP & $990 \mathrm{pb}^{-1}$ \\
Charm MC & RAPGAP & $990 \mathrm{pb}^{-1}$ \\
Light-flavour MC & ARIADNE & $990 \mathrm{pb}^{-1}$ \\
\hline
\end{tabular}

- MCs comprise LO matrix-elements with DGLAP parton showers

- Shapes are described but not normalisation

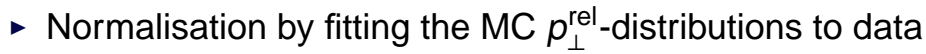




\section{Event Selection in Detail}

Pre-selection:

- All good events

- No explicit trigger

Vertex:

- $-50<Z_{\mathrm{vtx}}<50 \mathrm{~cm}$

Electron finder:

- $\mathcal{P}>0.9$

- $E_{\mathrm{e}}>10 \mathrm{GeV}$

- $Q_{\mathrm{el}}^{2}>4 \mathrm{GeV}^{2}$

- $y_{\mathrm{el}}<0.7$

- $y_{\mathrm{JB}}>0.05$
EFOs:

- $40<\left(E-p_{z}\right)<65 \mathrm{GeV}$

- $p_{\perp}<10 \mathrm{GeV}$

Calorimetry:

- $E_{\perp}-10^{\circ}$ cone $\geq 10 \mathrm{GeV}$

Tracking:

- $N_{\text {trk }}>8$

$p_{\perp}^{\text {rel }}$ calculation:

- use all jets with $E_{\perp}^{\text {jet }}>5 \mathrm{GeV}$ 


\section{Event Selection in Detail}

$\mu$ finding:

- $p_{\perp}^{\mu} \geq 1.5 \mathrm{GeV}$

- GMuon quality > 4

- Forward track muon quality modification

- $\mu$ regions

- forward: $1.2<\eta$

- barrel: $-0.9<\eta<1.2$

- rear: $-1.6<\eta<-0.9$

- HAC2 (rear) >0.3 GeV and no MV

- HAC2 (forward) > $0.4 \mathrm{GeV}$
Jets:

- $\geq 1$ jet found with $k_{\perp}$-clustering in $E$ recombination scheme (massive mode, 3211), w/o DIS electron EFO and

- $-2<\eta<2.5$

- $E_{\perp}^{\mathrm{lab}}>5 \mathrm{GeV}$

- $p_{\text {jet }}-p_{\mu}>0.7 \mathrm{GeV}$ anti-isolation 


\section{$\mu$-Efficiency Corrections}

- $\mu$-efficiencies differences between data and MC

- Correction factors obtained by independend $J / \psi$, Bethe-Heitler data-sets

- Binned in $p_{\perp}^{\mu}$ and $\eta^{\mu}$

- Efficiencies/inefficienies combined for all $\mu$-detectors

- MC $\mu$ weighted with combined correction factors

- Significant improvement of $\mu$ description

- Half of the correction assigned as syst. error $( \pm 10 \%)$ 


\section{Previous Results of Beauty in DIS}

\section{ZEUS}
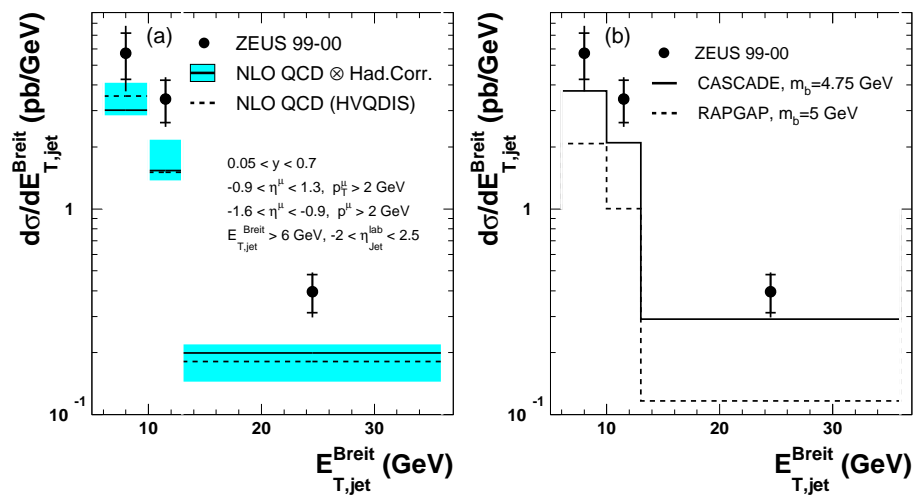


\section{Previous Results of Beauty in DIS}

\section{ZEUS}
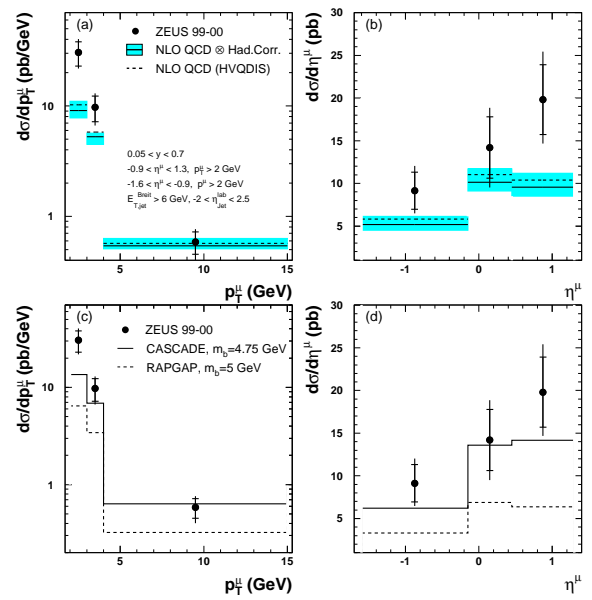


\section{Previous Results of Beauty in DIS}

\section{ZEUS}
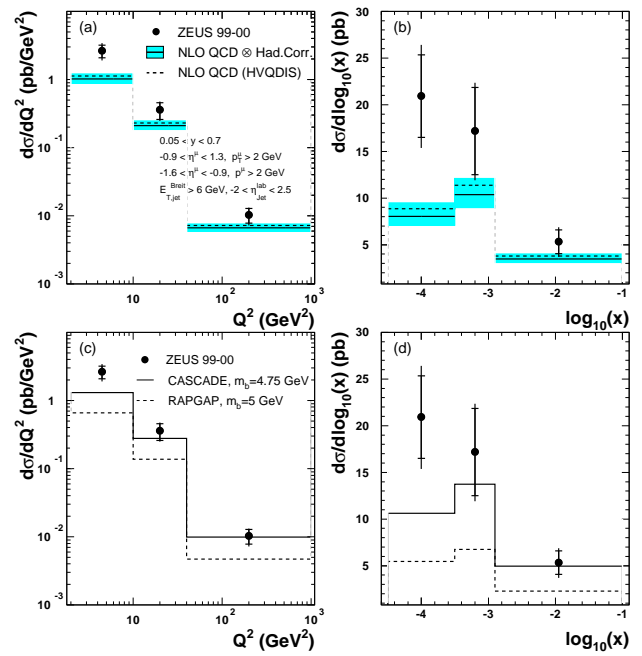